\title{
MOLECULAR DYNAMICS STUDY OF CRYSTALLINE WATER ICES
}

\author{
E. A. Zheligovskaya
}

UDC 539.21+546.212

The behavior of structures of $\mathrm{H}_{2} \mathrm{O}$ crystalline ices Ih, Ic, XI, VII, VIII, VI is studied in molecular dynamics experiment using the potential offered by Poltev and Malenkov. The behavior of the system consisting of one of the two identical interpenetrating, but without any common hydrogen bonds, water frameworks comprising the ice VI structure is also simulated. As a result of simulations, the ice VII structure has collapsed, whereas other systems proved to be stable. The reasons of instability of the ice VII and previously studied ice IV structures in molecular dynamics experiments are discussed. Based on the simulation results of the above-mentioned ices and previous simulation of ices II, III, IX, IV, and XII, the general regularities of dynamic properties of water molecules in crystalline water ices are formulated. Unreliability of results obtained by molecular dynamics in the investigation of self-organizing processes in aqueous systems is shown.

Keywords: aqueous systems, simulations, self-organization, molecular dynamics method, ices.

\section{INTRODUCTION}

Particular attention is paid at present to study the role of water in self-organizing processes in biological and synthetic supramolecular systems [1,2]. Only X-ray diffraction and high resolution electron microscopy (HREM) may be considered as direct experimental methods providing the study of these processes at the molecular level, their use meeting certain difficulties. So, even for crystal systems X-ray diffraction analysis does not always reveal their structure, and as for amorphous or liquid systems this method in principle can give only the average picture of a mutual arrangement of atoms in the form of radial density distribution functions. Images obtained by HREM are not very distinct and full of many random details; hence, their interpretation requires certain model representations of the systems under study. Thus, at present, the investigation of self-organizing process in a system at the molecular level imperatively requires the application of simulations, and for the adequate description of these processes it is necessary to take into account stereochemistry of molecules of the substances comprising the system under study.

The modular design method $[1,3,4]$ is the most direct method for simulating self-organizing processes in systems consisting of molecules with input stereochemical parameters (bond lengths, bond and torsion angles). A stability condition of structures formed in the course of self-organization is the absence of broken chemical bonds therein. The modular design method allows one to obtain structures with closed loops of bonds (i.e. the structures that do not contain broken bonds) from molecules with input stereochemical parameters and also to find possible ways to transform these structures into the other with closed loops of bonds with preserving the values of stereochemical parameters in the set interval. Thus possible routes of self-organization can be defined.

A. N. Frumkin Institute of Physical Chemistry and Electrochemistry, Russian Academy of Sciences, Moscow; lmm@phyche.ac.ru. Translated from Zhurnal Strukturnoi Khimii, Vol. 49, No. 3, pp. 476-489, May-June, 2008. Original article submitted September 4, 2007. 
The construction of structures with closed loops of bonds from molecules with input stereochemical parameters is a complex geometrical problem. In the case of water molecules, the closing conditions of bond loops are most difficult because each water molecule in the sought structure should form precisely four hydrogen bonds that must be directed almost exactly to vertices of a regular tetrahedron. Therefore in $[3,4]$ it was proposed to distinguish three-dimensional closed loops of bonds (these loops were called modules) in the structures of crystalline aqueous ices Ih and Ic formed under atmospheric pressure, then to transform them by the dispiration procedure (when only torsion angles are somewhat affected, whereas other parameters vary only slightly), and finally, to build the corresponding structures from these transformed three-dimensional modules that also do not contain broken bonds. Such a procedure of constructing a structure from crystalline and dispirationtransformed modules by their combination through common elementary cycles of bonds was called the modular design method [3,4]. Using this method, hydrate shells of various biological molecules [5], crystalline approximation of the structure of the surface layer (SL) of water at the interface with the gas phase, and the sequence of SL structural transformations with the formation and subsequent compression of a Langmuir monolayer on the SL [6] were constructed from the dispiration-transformed modules of ice Ih. From the dispiration-transformed modules of silicon (which is isostructural to diamond and ice Ic), the model for the structure of amorphous silicon inclusions [7] was constructed. This model seems to be applicable also to the description of structural elements of aqueous low density amorphous ice (LDAI) because the radial distribution functions of $\mathrm{Si}$ atoms in amorphous silicon and $\mathrm{O}$ atoms in LDAI coincide when the distance between atoms is expressed in units of the average separation between the nearest neighbors [8].

Difficulty in the application of the modular design method tempts to use simpler methods to simulate the selforganizing processes in which it would be enough to set some initial conditions, while the subsequent evolution of system would be described by a solution of some equations. Molecular dynamics, wide-spread at present, belongs to these methods. It requires the determination of the interaction potential between molecules, the initial (coordinates and velocities) and the boundary conditions. The system evolution is described then by the numerical solution of Newton equations of motion. In this case the reproduction of values of stereochemical parameters for chemical (including hydrogen) bonds between molecules depends on the correctness of the potential describing their interaction.

Thus, for an adequate description of the system evolution by molecular dynamics it is necessary: (1) to define the potential describing well the interaction of molecules with each other; (2) to set "reasonable" initial conditions from which the system could reach in principle the sought final state; and (3) to monitor a rather long trajectory of the system evolution during which the system would be self-organized into the sought final state with a definite structure.

All the above requirements can be satisfied now. Firstly, there is a large number of potentials that reproduce well various physical properties of water [9]. Secondly, the requirement of reasonable initial conditions is critical, as a rule, for simulating systems with polymeric molecules where transitions from one conformation to another can be hindered, and in the case of purely aqueous systems the configurations comprised by small water molecules can be easily rearranged if the temperature is not very low. At last, thirdly, modern computer calculations can cover time intervals up to several hundreds of nanoseconds [10]. Nevertheless, the analysis of published simulations of aqueous systems shows that the results of molecular dynamics simulations of self-organizing processes in aqueous systems should be considered critically.

In the present paper we analyze the behavior of 11 structures of known now 15 crystalline modifications of water ice $[11,12]$ using molecular dynamics simulations with different interaction potentials and also the results of simulations of crystallization processes in bulk water without artificial introduction of the crystallization center [10] and of crystallization at the crystal ice-melt interface [13]. It is shown that in computer experiments both the stability of systems with the known structure and the process of structure self-organization can differ from the corresponding results of real physical experiments.

Results of molecular dynamics simulations of ices II and IX [14, 15], III and IX [16], and IV and XII [17] with use of the potential offered by Poltev and Malenkov (further the PM potential) have been presented in the previous papers where the dynamic characteristics of water molecules in these ices were considered. The results of simulations of $\mathrm{H}_{2} \mathrm{O}$ ices Ih, Ic, XI, VII, VIII, and VI using the PM potential are presented here. Simulations of ices Ih, Ic, II-IX, and XI with the use of $\mathrm{SPC} / \mathrm{E}$ and TIP4P potentials are considered in the works of other authors. 


\section{CONDITIONS FOR SIMULATION OF CRYSTAL WATER ICES}

Table 1 summarizes the unit cell parameters, the density and degree of proton ordering of crystalline water ices, and references to the works in which their structures are described.

In order to simulate crystalline water ices the rectangular simulation boxes with periodic boundary conditions were used. Table 2 lists the parameters of simulation boxes. In the case of ices Ic, XI, VII, VIII, and VI, each simulation box contained an integer number of their rectangular unit cells along each axis. In the structure of ice Ih with a hexagonal unit cell the smallest periodically repeated rectangular cell was distinguished (its dimensions were $a \times 3^{1 / 2} a \times c$, where $a$ and $c$ are the unit cell parameters), and the simulation box containing $6 \times 3 \times 3$ of these rectangular cells was used. The initial coordinates of atoms are obtained from the crystallographic data given in the corresponding references (Table 1). To simulate protondisordered ices the hydrogen atoms were placed into possible positions in a simulation box, according to the Bernal-Fowler rules, using the algorithm proposed in [24]. It was specially verified that the population of all positions was $1 / 2$ and the total dipole moment of the simulation box was zero.

Interactions between $\mathrm{O}$ and $\mathrm{H}$ atoms of water molecules were described using the PM potential [25]. Within the limits of this modeling potential it is supposed that the water molecule is rigid with the fixed bond angle $\mathrm{HOH}$ equal to the tetrahedral angle $\operatorname{arc} \cos (-1 / 3) \approx 109.5^{\circ}$, and with the $\mathrm{OH}$ bond length of $0.98 \AA$. The electric charge of $-0.68 \mathrm{e}$ is placed on the oxygen atom, and the $+0.34 \mathrm{e}$ charge is placed on each hydrogen atom. Interactions between $\mathrm{O}$ and $\mathrm{O}, \mathrm{O}$ and $\mathrm{H}, \mathrm{H}$ and $\mathrm{H}$ atoms belonging to different water molecules are described by the potential similar to the Lennard-Jones potential plus the electrostatic term

$$
U=\sum_{i j}\left(k q_{i} q_{j} / r_{i j}-A_{i j} / r_{i j}^{n}+B_{i j} / r_{i j}^{12}\right)
$$

TABLE 1. Crystallographic Parameters of the Structures of Crystalline Ices

\begin{tabular}{|c|c|c|c|c|c|c|c|c|}
\hline Ice & Substance & Space group & $T, P^{*}$ & $\begin{array}{c}\text { Unit cell type } \\
\text { and } \\
\text { parameters }\end{array}$ & $Z^{* *}$ & $\rho, \mathrm{g} / \mathrm{cm}^{3 * * *}$ & $\begin{array}{c}\text { Proton } \\
\text { ordering }\end{array}$ & Ref. \\
\hline Ih & $\mathrm{D}_{2} \mathrm{O}$ & $P 6_{3} / m m c$ & $\begin{array}{l}223 \mathrm{~K} \\
1 \mathrm{Bar}\end{array}$ & $\begin{array}{c}\text { Hexagonal } \\
a=4.513 \AA \\
c=7.355 \AA\end{array}$ & 4 & $\begin{array}{c}1.03 \\
(0.92)\end{array}$ & $\begin{array}{c}\text { Totally } \\
\text { disordered }\end{array}$ & {$[18]$} \\
\hline Ic & $\mathrm{D}_{2} \mathrm{O}$ & $F d \overline{3} m$ & $\begin{array}{l}177 \mathrm{~K} \\
1 \mathrm{Bar}\end{array}$ & $\begin{array}{c}\text { Cubic } \\
a=6.353 \AA\end{array}$ & 8 & $\begin{array}{c}1.04 \\
(0.93)\end{array}$ & $\begin{array}{c}\text { Totally } \\
\text { disordered }\end{array}$ & [19] \\
\hline $\mathrm{XI}$ & $\mathrm{D}_{2} \mathrm{O}$ & $\mathrm{Cmc} 2_{1}$ & $\begin{array}{l}5 \mathrm{~K}, \\
1 \mathrm{Bar}\end{array}$ & $\begin{array}{c}\text { Rhombic } \\
a=4.5019 \AA, \\
b=7.7978 \AA, \\
c=7.3280 \AA\end{array}$ & 8 & $\begin{array}{c}1.03 \\
(0.93)\end{array}$ & Ordered & [20] \\
\hline VII & $\mathrm{D}_{2} \mathrm{O}$ & $\operatorname{Pn} \overline{3} m$ & $\begin{array}{c}295 \mathrm{~K}, \\
2.6 \mathrm{GPa}\end{array}$ & $\begin{array}{c}\text { Cubic } \\
a=3.3501 \AA\end{array}$ & 2 & $\begin{array}{c}1.77 \\
(1.59)\end{array}$ & $\begin{array}{c}\text { Totally } \\
\text { disordered }\end{array}$ & [21] \\
\hline VIII & $\mathrm{D}_{2} \mathrm{O}$ & $I 4_{1} /$ amd & $\begin{array}{c}269 \mathrm{~K}, 2.8 \\
\mathrm{GPa}\end{array}$ & $\begin{array}{c}\text { Tetragonal } \\
a=4.6779 \AA, \\
c=6.8029 \AA\end{array}$ & 8 & $\begin{array}{c}1.79 \\
(1.61)\end{array}$ & $\begin{array}{l}\text { Totally } \\
\text { ordered }\end{array}$ & [22] \\
\hline VI & $\mathrm{D}_{2} \mathrm{O}$ & $P 4_{2} / n m c$ & $\begin{array}{l}225 \mathrm{~K}, \\
1.1 \mathrm{GPa}\end{array}$ & $\begin{array}{c}\text { Tetragonal } \\
a=6.1812 \AA, \\
c=5.6980 \AA\end{array}$ & 10 & $\begin{array}{c}1.53 \\
(1.37)\end{array}$ & $\begin{array}{c}\text { Totally } \\
\text { disordered }\end{array}$ & [23] \\
\hline
\end{tabular}

*Temperature and pressure at which the structure was determined.

**The number of water molecules in the unit cell.

$* * *$ The value calculated for $\mathrm{H}_{2} \mathrm{O}$ ices at the same values of structural parameters is presented in parentheses. 
TABLE 2. Conditions of the Numerical Simulation Experiment for the Behavior of Crystalline Ices

\begin{tabular}{c|c|c|c|c|c}
\hline Ice & $A, B, C, \AA$ & $N$ & $K$ & $E, \mathrm{kcal} / \mathrm{mol}$ & $T, \mathrm{~K}$ \\
\hline Ih & $27.078,23.450,22.065$ & 432 & 108 & -12.1 & 58 \\
Ic & $25.412,25.412,25.412$ & 512 & 64 & -12.1 & 61 \\
XI & $27.0114,23.3934,21.9840$ & 432 & 54 & -11.0 & 61 \\
VI & $24.7248,24.7248,22.792$ & 640 & 10 & -11.5 & 82 \\
VI' & $24.7248,24.7248,22.792$ & 320 & 10 & -11.0 & 47 \\
VII & $20.1006,20.1006,20.1006$ & 432 & 216 & -11.5 & 20 \\
VIII & $18.7116,18.7116,22.7920$ & 512 & 64 & -11.0 & 40
\end{tabular}

Note. $A, B, C$ are the dimensions of the rectangular simulation box used; $N$ is the number of water molecules in the simulation box; $K$ is the equivalent number of unit cells; $E$ is the total energy of the system; $T$ is the temperature; VI' is the system formed by one of the two identical independent interpenetrating aqueous frameworks of the ice VI structure.

Here $q_{\mathrm{i}}$ and $q_{\mathrm{j}}$ are the charges on $i$ and $j$ atoms; $r_{i j}$ is the distance between $i$ and $j$ atoms expressed in $\AA ; k=332$ is the conversion coefficient to express the energy in $\mathrm{kcal} / \mathrm{mol} ; A_{i j}$ and $B_{i j}$ are the empirical parameters $\left(A_{\mathrm{OO}}=200, B_{\mathrm{OO}}=410,000\right.$, $A_{\mathrm{OH}}=3700, B_{\mathrm{OH}}=9700, A_{\mathrm{HH}}=40, B_{\mathrm{HH}}=3800$ for the energy expressed in $\mathrm{kcal} / \mathrm{mol}$ and distances $r_{i j}$ in $\AA$ ); $n=6$ for the interactions between $\mathrm{O}$ and $\mathrm{O}$ or $\mathrm{H}$ and $\mathrm{H}$ atoms, and $n=10$ for the interactions between $\mathrm{O}$ and $\mathrm{H}$. Simulations with the given potential reproduce well, in particular, the values of the self-diffusion coefficient of water molecules in supercooled water at different temperatures and pressures [9].

In the simulations, the $N V E$ ensemble was used, where $N$ is the number of molecules in the system, $V$ is the volume of the system, and $E$ is its total energy. The value of $E$ was set. The temperature $T$ of the modeled system was then calculated as the time averaged kinetic energy of the system per a degree of freedom. Table 2 summarizes the values of $E$ and the corresponding values of $T$ at which simulations were performed. An integration step of $1 \mathrm{fs}=10^{-15} \mathrm{~s}$ was chosen. Other details of molecular dynamics simulations of ices are presented in the works [14-17].

Stability of the ice structures during simulation was controlled both visually, using the snapshots of the system under study, and by the analysis of the hydrogen-bond network. For this purpose a geometrical criterion of the hydrogen bond was used, according to which the hydrogen bond between two water molecules does exist if the OO separation is less than $3.2 \AA$ and any of the distances between the $\mathrm{O}$ atom of one of the molecules and $\mathrm{H}$ atom of another molecule is less than $2.4 \AA$. These boundary values are slightly smaller than those used for the analysis of hydrogen bonds in liquid water under normal conditions (3.3 $\AA$ and $2.6 \AA$ [26] respectively), but they are large enough to describe hydrogen bonds in crystalline ices [15].

For ices with the structures not collapsed during molecular dynamic simulations, the following characteristics were calculated: the average square of the mass center shift of a water molecule

$$
\left\langle\boldsymbol{r}^{2}(t)\right\rangle=\frac{1}{N} \sum_{i=1}^{N}\left(\boldsymbol{r}_{i}(t)-\boldsymbol{r}_{i}(0)\right)^{2},
$$

where $\boldsymbol{r}_{i}(t)$ is the radius-vector of the mass center of the $i$ th water molecule at the time $t$; summation is performed over all $N$ molecules in the simulation box; the velocity autocorrelation function of the mass center of a water molecule

$$
c(t)=\frac{1}{N} \sum_{i=1}^{N}\left(\boldsymbol{v}_{i}(t) \boldsymbol{v}_{i}(0) / \frac{1}{N} \sum_{i=1}^{N}\left(\boldsymbol{v}_{i}(0) \boldsymbol{v}_{i}(0)\right),\right.
$$

where $v_{i}(t)$ is the velocity of the mass center of the $i$ th water molecule at the time $t$, and the velocity autocorrelation function of hydrogen atoms $c_{\mathrm{H}}(t)$.

Spectra of translational vibrations of water molecules were obtained by the Fourier transformation of the corresponding velocity autocorrelation function of the mass center of water molecules

$$
\varphi(\omega)=\int_{0}^{\infty}(\cos \omega t)(\langle\boldsymbol{v}(t) \boldsymbol{v}(0)\rangle /\langle\boldsymbol{v}(0) \boldsymbol{v}(0)\rangle) d t,
$$


and librational spectra were obtained by the Fourier transformation of the corresponding velocity autocorrelation function of hydrogen atoms. Of course, the vibrational spectra of hydrogen atoms contain the bands corresponding to translational vibrations.

\section{RESULTS OF SIMULATION OF CRYSTALLINE WATER ICES. Ices Ih, Ic, XI, VII, and VIII}

Description of the ice Ih, Ic, XI, VII, and VIII structures. Ices Ih, Ic, and XI formed under atmospheric pressure and ices VII and VIII formed under a pressure of $2 \mathrm{GPa}$ up to $60 \mathrm{GPa}$ have relative structures.

Ice Ih, thermodynamically stable at normal pressure, and metastable ice Ic are characterized by the absence of proton ordering. Their structures are related in the same way as the lonsdaleite and diamond or wurtzite and sphalerite structures (Fig. 1 $a, b$ ). The structures of ices Ih and XI have an identical oxygen framework, but unlike ice Ih, in ice XI protons are orderly located (see Fig. 1a,c). Thus, in ices Ih, Ic, and XI oxygen atoms of the nearest neighbors of any water molecule are located in vertices of an almost regular tetrahedron.

There are two crystallographically independent oxygen atoms in the ice XI structure. Water molecules with atom $\mathrm{O}(1)$ are oriented in such a manner that one of their hydrogen atoms $(\mathrm{H}(3))$ forms bonds between hexagonal layers, while both hydrogen atoms (depicted as $\mathrm{H}(5)$ ) of molecules with atom $\mathrm{O}(2)$ form bonds inside the hexagonal layer (Fig. 1c). In the ice Ih structure the positions of all oxygen atoms are related by symmetry transformations. Still, it is also possible to distinguish the same two types of orientations of water molecules there: $(\mathrm{H}(2) \mathrm{O}(1) \mathrm{H}(3)$ and $\mathrm{H}(3) \mathrm{O}(1) \mathrm{H}(3)$, Fig. $1 a$.

The ice VII and VIII structures can both be considered as two ice Ic structures inserted into each other; in ice VII protons are disordered, whereas ice VIII is characterized by complete proton ordering. The ice VII structure is cubic (see Fig. 1d). In the first approximation one may consider that in this structure $\mathrm{O}$ atoms occupy node positions in the bbc lattice. In the ice VIII structure $\mathrm{H}$ atoms are located in such a manner that each of two identical independent water frameworks has a nonzero dipole moment (see Fig. 1e). Owing to electrostatic interaction, the frameworks are displaced relative to each other and, unlike ice VII with the cubic structure, the structure of ice VIII is tetragonal.

Stability of the structures of ices Ih, Ic, XI, VII, and VIII in the molecular dynamics experiment with the Poltev-Malenkov potential. The snapshots and the analysis of hydrogen bonds in modeled ices Ih, Ic, XI, VII, and VIII prove that during molecular dynamics simulations with the PM potential the structures of ices Ih, Ic, XI, and VIII do not collapse, whereas the ice VII structure does break almost in the very beginning of simulation.

As mentioned above, the densities of ices VII and VIII are very close and their structures are very similar. In the ice VII structure each oxygen atom has eight nearest neighboring $\mathrm{O}$ atoms at a distance of about $2.90 \AA$; it forms a hydrogen bond with four of them, whereas the rest four neighboring $\mathrm{O}$ atoms belong to the other water framework, i.e., they are not hydrogen-bonded with the $\mathrm{O}$ atom under consideration [21,23]. Alternatively, in the ice VIII structure each $\mathrm{O}$ atom has two of four not bonded with it neighboring $\mathrm{O}$ atoms at a larger $(3.32 \AA)$ and two other at a smaller $(2.74 \AA)$ distance than the lengths of hydrogen bonds with yet another four $\mathrm{O}$ atoms $(2.89 \AA)$ [22, 23]. Nevertheless, as the ice VIII structure does not collapse during simulation, the larger density and the presence of close but not hydrogen-bonded oxygen atoms cannot serve as a direct cause of the destruction of the ice VII structure.

A basic distinction between ices VII and VIII is the proton ordering of ice VIII. Because of this, in the ice VIII structure the positions of oxygen atoms are strictly determined and well resolved by structural analysis methods, whereas in the ice VII structure they are scattered around the positions of bbc lattice nodes. Some assumptions on the character of disorder in positions of $\mathrm{O}$ atoms in the ice VII structure are analyzed in the work [27].

As the exact positions of $\mathrm{O}$ atoms in the ice VII structure are still unknown, the average positions of $\mathrm{O}$ atoms in the nodes of the bbc lattice were taken as the initial coordinates to simulate this ice. Relaxation of the given structure at zero temperature to the minimum of the potential energy, determined by the PM potential, led only to an insignificant (about the 

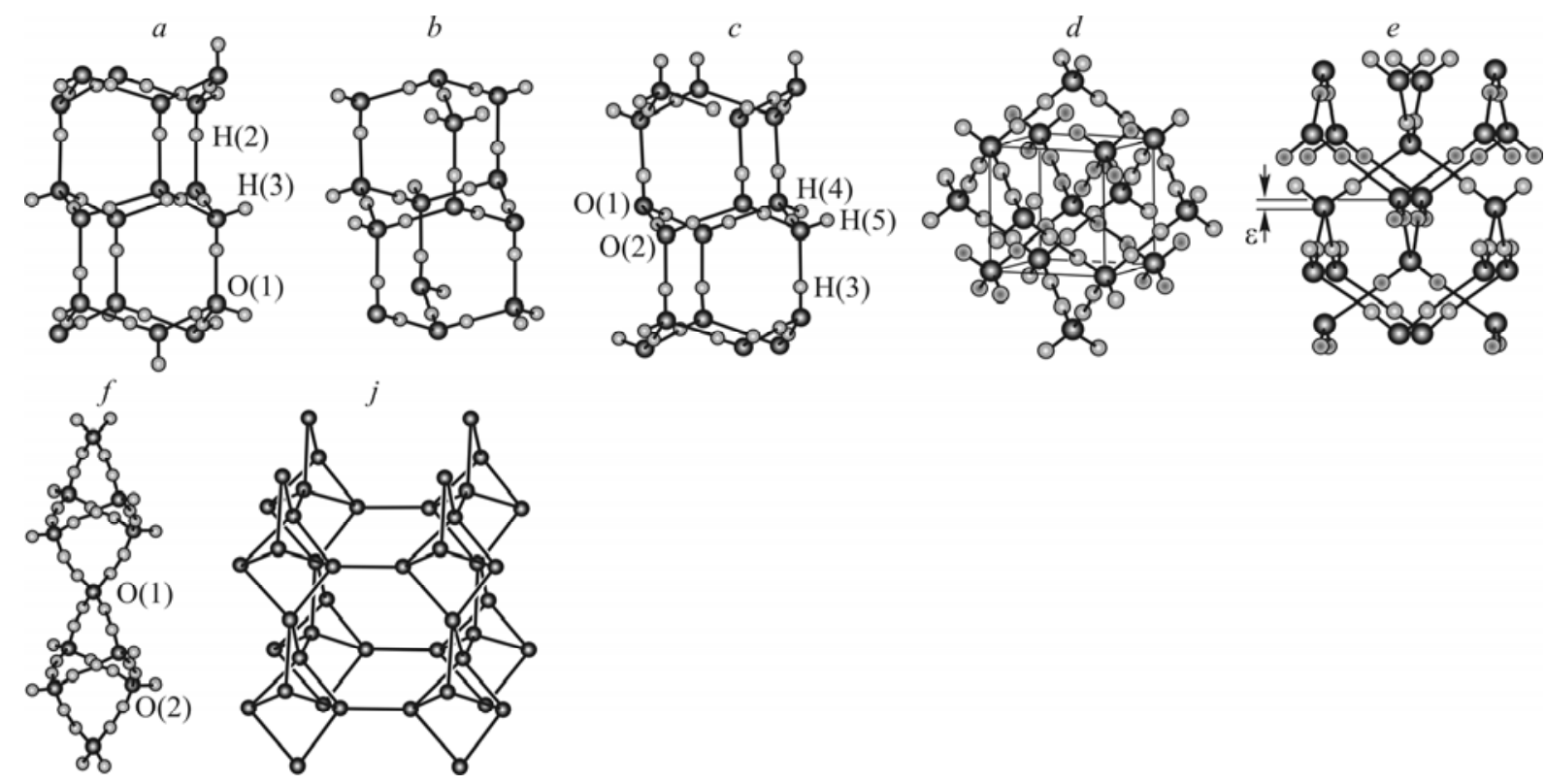

Fig. 1. Structures of ices Ih, Ic, XI, VII, VIII, and VI. Structure fragments of proton-disordered ices Ih $(a)$ and Ic (b) with a possible arrangement of $\mathrm{H}$ atoms; a fragment of the proton-ordered ice XI structure $(c)$. A fragment of the ice VII structure $(d)$; all possible positions of $\mathrm{H}$ atoms are displayed, their population is 0.5 ; the unit cell is designated. A fragment of the ice VIII structure (e); the shift $\varepsilon$ of independent frameworks relative to each other is shown. In Figs. $d$ and $e \mathrm{H}$ atoms of one of the two identical independent Ic water frameworks forming structures VII and VIII are blacked out. A basic element of the ice VI structure is a chain of water molecules stretched along the $c$ axis $(f)$; all possible positions of $\mathrm{H}$ atoms are shown. Association of chains into one of the two identical independent water frameworks forming the ice VI structure $(g)$; the chains forming the second framework pass through the channels of this framework; positions of $\mathrm{H}$ atoms are not designated.

order of several hundredths of Angstrom) shift of all atoms not causing the destruction of this structure. However, all attempts to carry out further molecular dynamics simulation of ice VII at a nonzero temperature resulted in the destruction of this system.

The PM potential, including, in particular, the rigid model of water molecule, appears to insufficiently correctly describe some fine features of the interaction between water molecules; so the potential energy minimum determined by it for the ice VII structure turns out to be unstable. Note that a change in the fixed bond angle $\mathrm{HOH}$ from tetrahedral to $104^{\circ}$ in the PM potential (like in $\mathrm{D}_{2} \mathrm{O}$ molecules in the ice VIII structure $[22,23]$ ) does not lead to stability of the ice VII structure. Another reason for instability of the ice VII structure is also theoretically possible, namely, locally unsuccessful arrangement of hydrogen atoms in some regions of the structure which leads to local instability of these regions that start the destruction of the whole structure at a nonzero temperature. Therefore, simulations of ice VII for various random, but satisfying the BernalFowler's rules, arrangements of $\mathrm{H}$ atoms, has been performed; but in all cases the destruction of these structures was observed at a nonzero temperature.

Similarly, the destruction of the ice IV structure revealed previously [17] in the course of molecular dynamics simulation also cannot be explained by its high density $\left(1.272 \mathrm{~g} / \mathrm{cm}^{3}\right.$ in ice $\mathrm{IV} \mathrm{H}_{2} \mathrm{O}$ [28], i.e. approximately the same [29] as in non collapsing during simulation [17] ice XII, and is even lower than that in non collapsing ices VI and VIII). More exact determination of the ice IV structure [30] shows that positions of $\mathrm{O}(2)$ atoms in it are also scattered relative to previously found average $\mathrm{O}(2)$ positions. Nevertheless, relaxation of the ice IV structure with $\mathrm{O}(2)$ atoms in the average positions at zero temperature to a minimum of the potential energy determined by the PM potential also resulted only in an insignificant shift of all atoms without the destruction of the structure. It seems that, as in the case of ice VII, the cause of instability of the ice IV structure leading to its destruction at a nonzero temperature is the insufficient accuracy of the PM potential. The presence 
of six close (3.14 $\AA$ and $3.29 \AA[28]$ ), but non hydrogen-bonded with it $\mathrm{O}(1)$ atoms nearby each $\mathrm{O}(2)$ atom in the ice IV structure is not a direct reason, but it promotes the destruction of this structure.

The refinement of the interaction potential between water molecules is not a trivial problem because some hydrogenbonded structures can not be calculated at present even by quantum mechanical methods. So, according to recent calculations of the structures of crystalline water ices with the use of non empirical formalism PACHA based on the density functional method in the spherical approximation [31], the ice IV structure described in the work [28] should not exist at all due to its strong instability. Based on these calculations, even the "corrected" structure has been proposed for ice IV [31]. Meanwhile, neutron diffraction confirmed the correctness of the previous determination [28] of the ice IV structure in the work [30]. Note that calculations of the ice VII structure by the method [31] nevertheless reproduced the experimentally observed structure of this ice.

During simulation of the behavior of systems consisting of one independent water framework of the structures of ices VII and VIII (i.e. a separate framework of ice Ic with increased values of lattice parameters at the same value of $\mathrm{OH}$ bond lengths in water molecules and, in the case of ice VIII, with proton ordering), the structures of these systems collapsed. This is not surprising because the framework of the Ic type is rather loose, and for its stabilization at high pressures additional molecules should occupy its interstices that, in the case of ices VII and VIII, create another water framework. The framework of the Ic type can be stabilized not only by water molecules. So, at pressures of $2.3 \mathrm{GPa}$ up to at least $30 \mathrm{GPa}$, gas hydrate with a water framework of the ice Ic structure forms in the system $\mathrm{H}_{2}-\mathrm{H}_{2} \mathrm{O}$. Its interstices, i.e. positions of $\mathrm{O}$ atoms of another independent water framework of VII ice, are occupied by $\mathrm{H}_{2}$ molecules [32].

Dynamic properties of water molecules in ices Ih, Ic, XI, and VIII. As expected, dynamic characteristics of water molecules in ices Ih and Ic almost do not differ (Fig. 2a, b) because they are determined mainly by the nearest environment of water molecule that is the same in both ices. A small distinction in the average square of the mass center shift of water molecule in ices Ih and XI seems to be caused by a small difference in the structure of their oxygen frameworks.

Translational and librational spectra of water molecules of ice XI contain much sharper peaks than the corresponding spectra of its proton-disordered structural analogue, ice Ih. This is so because water molecules containing oxygen atoms of the same crystallographic type are in identical potential wells in the structure of proton-ordered ice XI, while in the ice Ih structure, due to disorder in the arrangement of protons, potential wells of all water molecules somewhat differ (though all $\mathrm{O}$ atoms are crystallographically indiscernible in this structure), which leads to strong broadening of peaks.

Librational spectra of variously oriented water molecules differ significantly in ice XI (see Fig. 2b), while in ice Ih they almost coincide. Note that in the other pair of crystal ices with an identical oxygen framework (III and IX) dynamic characteristics of molecules with crystallographically independent $O$ atoms differ more strongly in proton-ordered ice IX than in proton disordered ice III [16].

As one would expect, dynamic characteristics of molecules in different water frameworks of ice VIII turned out to be identical, therefore the corresponding curves in Fig. 3 are presented only for the system on the whole. Vibrational spectra of ice VIII contain many sharp peaks, as is should be for proton-ordered ice.

Since the interaction between independent water frameworks in the ice VIII structure is rather strong (one framework collapses in the absence of the second one), and moreover, the frameworks of the ice VIII structure are protonordered and the distances between their molecules are longer than in the ice Ic structure, vibrational spectra of ices VIII and Ic strongly differ.

\section{Ice VI and the System of One of the Two Constituent Identical Independent Water Frameworks}

Ice VI is thermodynamically stable at a pressure of $0.6 \mathrm{GPa}$ up to $2 \mathrm{GPa}$ and consists of two identical interpenetrating independent water frameworks (Fig. $1 f, g$ ); each of them is the structural analogue of a silica-alumina framework ( $\mathrm{Al}, \mathrm{Si}) \mathrm{O}_{2}$ in the mineral edingtonite [23]. Chemical bonds (including hydrogen bonds) between independent water frameworks constituting ice VI are absent, but each of them, occupying interstices of another framework, renders a 
$a$

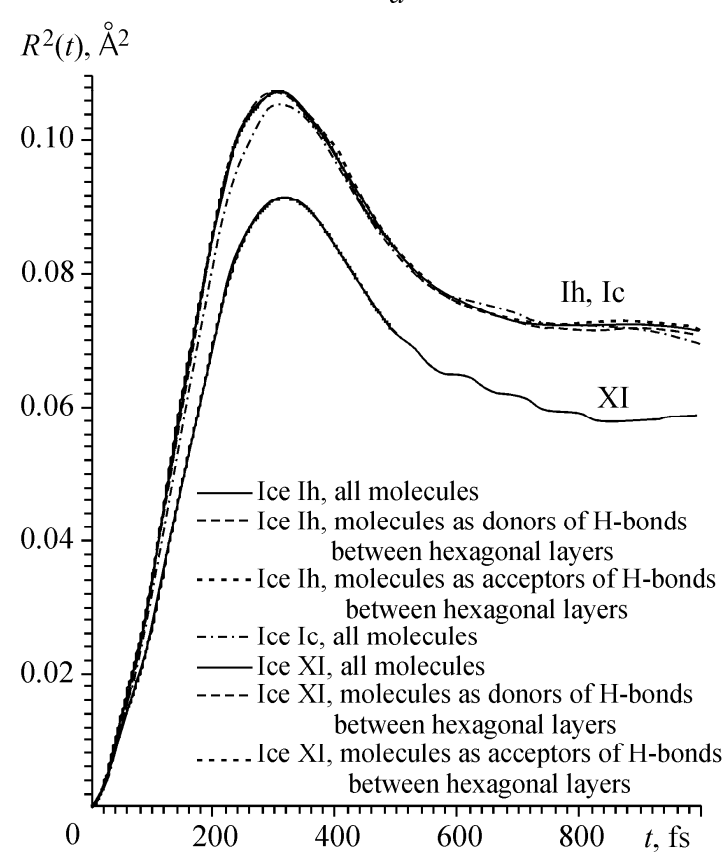

$b$

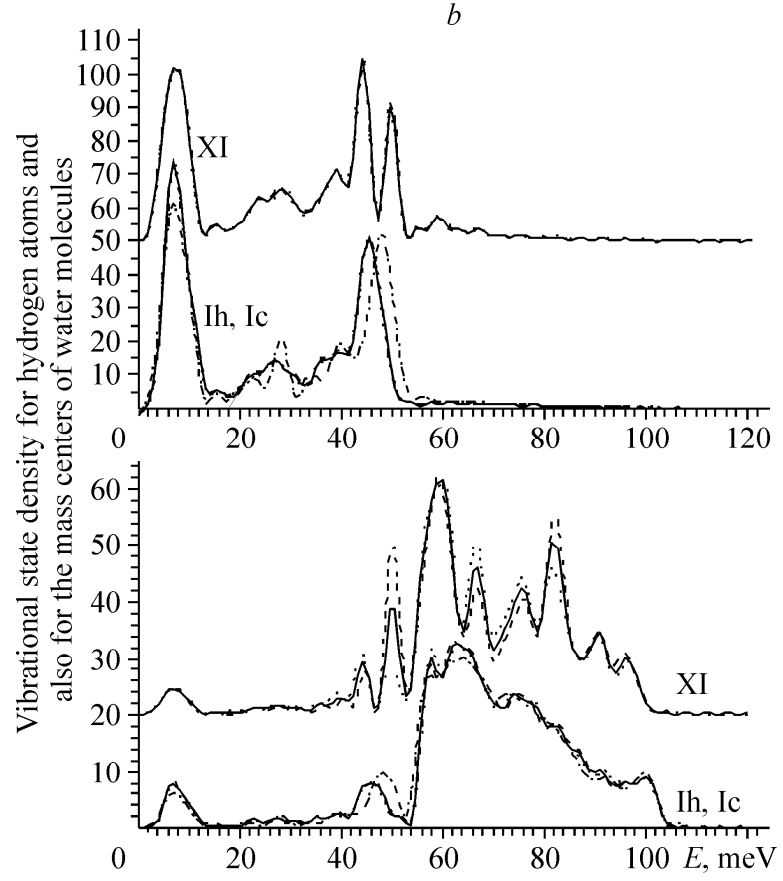

Fig. 2. Average square of the mass center shift of water molecules in ice Ih at $58 \mathrm{~K},(a)$ : Solid line, all water molecules; dashed line, $\mathrm{H}(2) \mathrm{O}(1) \mathrm{H}(3)$ molecules; dotted line, $\mathrm{H}(3) \mathrm{O}(1) \mathrm{H}(3)$ molecules; Ic at $61 \mathrm{~K}$ (dot-and-dash line, all water molecules) and XI at $61 \mathrm{~K}$ (solid line, all molecules; dashed line, $\mathrm{H}(3) \mathrm{O}(1) \mathrm{H}(4)$ molecules; dotted line, $\mathrm{H}(5) \mathrm{O}(2) \mathrm{H}(5)$ molecules. Curves for variously oriented water molecules in ice $\mathrm{Ih}(\mathrm{H}(2) \mathrm{O}(1) \mathrm{H}(3)$ and $\mathrm{H}(3) \mathrm{O}(1) \mathrm{H}(3))$ or in ice $\mathrm{XI}(\mathrm{H}(3) \mathrm{O}(1) \mathrm{H}(4)$ and $\mathrm{H}(5) \mathrm{O}(2) \mathrm{H}(5))$ almost coincide. Density of vibrational states of water molecules in ices Ic and XI, $(b)$ : Designations of curves are the same as in Fig. $a$. Curves for variously oriented water molecules in ice Ih almost coincide. Spectra of ice XI are shifted along the $y$ axis for convenience. Translational vibrations are at top, librations are below.
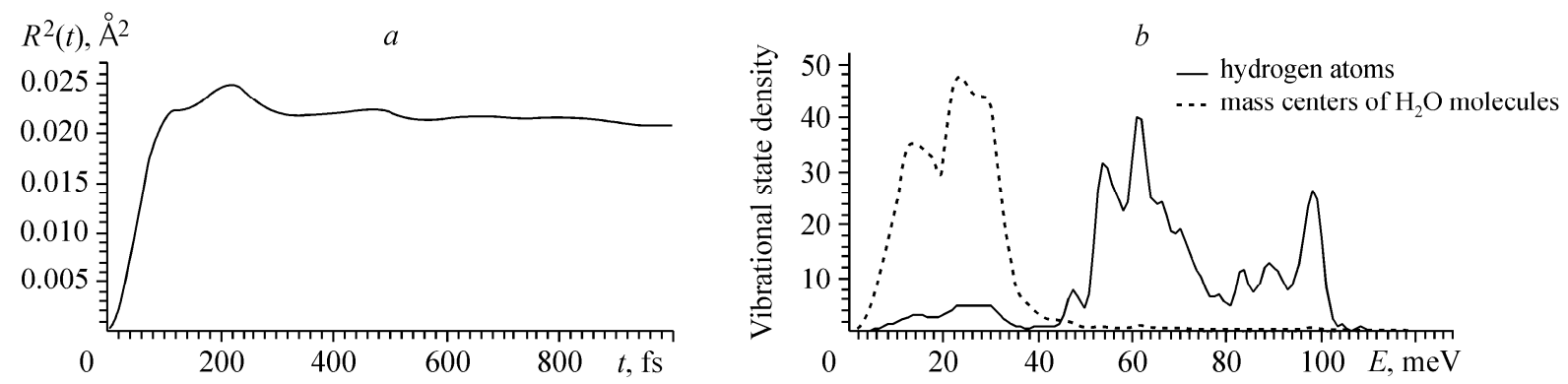

Fig. 3. Average square of the mass center shift of water molecule in ice VIII at $40 \mathrm{~K}$ : (a) Density of vibrational states of water molecule in ice VIII at $40 \mathrm{~K} ;(b)$ dashed line shows translational vibrations; solid line designates librations.

stabilizing effect on it. For this reason, ice VI (as well as ices VII and VIII) is called sometimes the self-clathrate. According to [23], positions of hydrogen atoms in the ice VI structure are occupied completely randomly.

During molecular dynamic simulations with the PM potential the ice VI structure did not collapse. Furthermore, we have simulated the system in one of the two identical independent water frameworks that form ice VI. It turned out that one independent framework, not stabilized by another framework, also represents a stable system not collapsing during simulations with the PM potential. Note that empty water frameworks of gas hydrates also keep their integrity in molecular dynamics simulation [33].

As the average potential energy of interaction with other water molecules is higher in the system consisting of one independent framework than in ice VI, the average kinetic energy of water molecule in the one framework system should be 

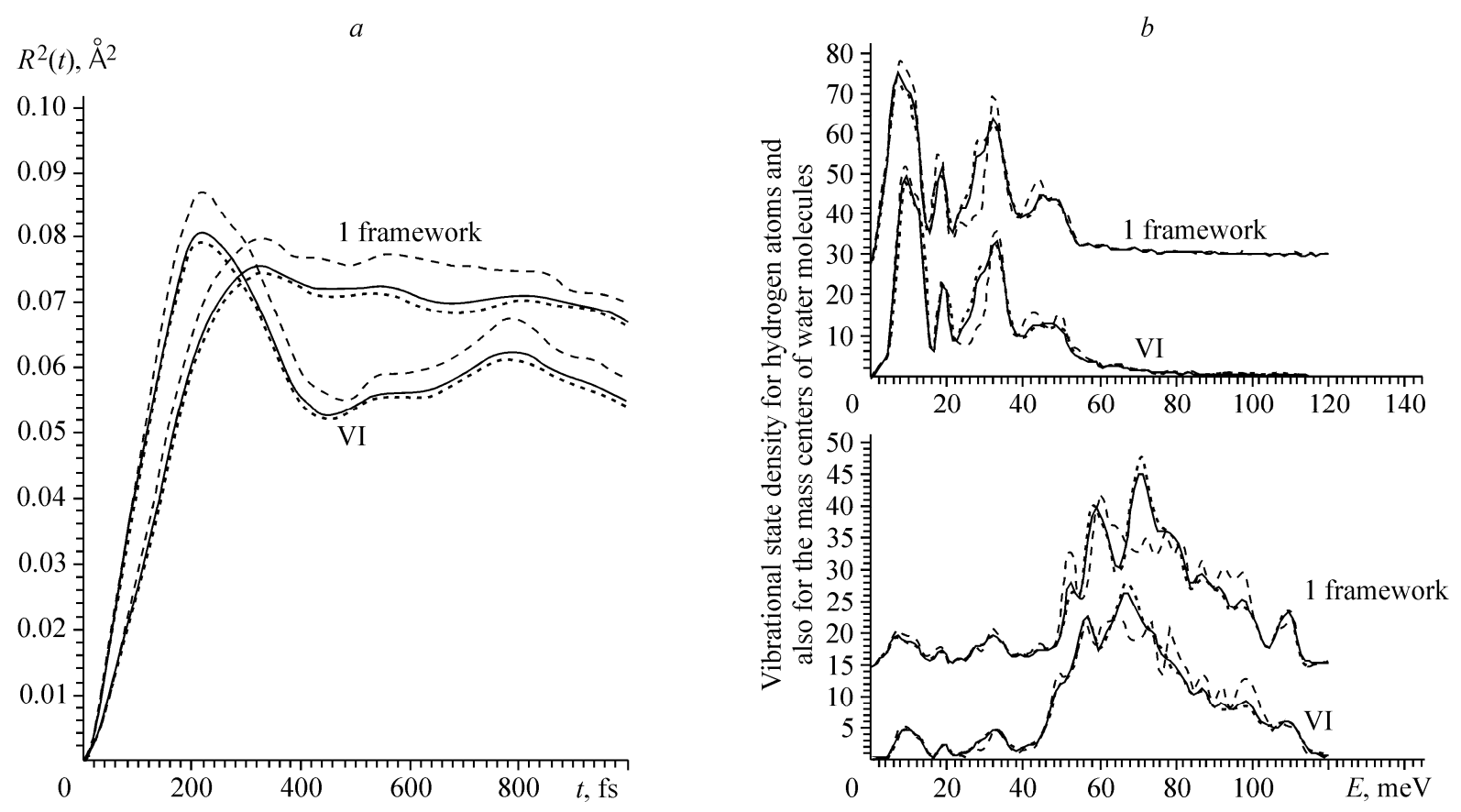

Fig. 4. (a) Average square of the mass center shift of water molecule in ice VI at $82 \mathrm{~K}$ and in the system consisting of one of the two independent water frameworks in the ice VI structure at $47 \mathrm{~K}$; $(b)$ Density of vibrational states of water molecules in ice VI and in the system of one of its two independent water frameworks. Solid line: all molecules; dashed line: $\mathrm{H}_{2} \mathrm{O}(1)$ molecules; dotted line: $\mathrm{H}_{2} \mathrm{O}(2)$ molecules; designations of oxygen atoms in ice VI are specified in Fig. $1 f$. The curves for the one framework system are shifted along the $y$ axis for convenience. Translational vibrations are at top, librations are below.

lower than in ice VI at the same total energy of the system $(E)$. Therefore even at a higher value of $E$ the temperature of the one framework system can be lower than in ice VI (Table 2). Fig. $4 a$ shows that at the same value of $E$ the amplitude of vibrations of water molecules is higher in the one framework system than in ice VI consisting of two frameworks.

The interaction of independent water frameworks of ice VI weakly affects the spectra of translational and librational vibrations of water molecules: they differ almost only by a small shift of librational peaks of molecules in the system with one independent framework in comparison with the spectrum of ice VI (Fig. 4b). A somewhat more blurred character of vibrational spectra of molecules in ice VI, as compared to the spectrum of the one framework system, can be caused by a higher simulation temperature for ice VI. Distinctions in vibrational spectra of molecules with $O(1)$ and with $O(2)$ atom (see designations of atoms in Fig. $1 f$ ) are also identical for ice VI and the system with one independent framework (Fig. 4b).

Note that the dynamic properties of water molecules in empty water frameworks of gas hydrates and in the same frameworks, but with the interstices filled by gas molecules, also almost do not differ [33]. This is explained by weak interaction of gas guest atoms with molecules of the water host framework, which is insufficient for a significant change in their vibrational spectrum. Experimentally this assumption is supported by that the spectra of neutron incoherent inelastic scattering (NIIS) in ice II with empty channels and in ice II with channels filled by helium atoms almost coincide [34]. However, in ice VI the shortest separations between oxygen atoms belonging to different frameworks and, hence, not forming hydrogen bonds with each other, are only 3.34-3.47 $\AA$ (while the hydrogen bond length in this ice under the same conditions varies from $2.72 \AA$ to $2.79 \AA$ and the distances to the second neighbors are 3.91- $4.96 \AA$ ) [23]. Therefore, though molecules of one framework do not belong to the nearest environment of molecules in another framework and the interaction between independent water frameworks of ice VI does not change significantly the general form of vibrational spectra of ice VI molecules, nevertheless it leads to a considerable shift of librational peaks in comparison with their position in the spectrum of one independent water framework system. 


\section{General Conclusions Following from the Results of Molecular Dynamics Simulations of Crystalline Ices Ih, Ic, XI, II [14, 15], III [16], IX [14-16], IV [17], XII [17], VI, VII, and VIII}

Based on the presented results and the data of previous works [14-17] it is possible to draw the following conclusions.

Structural stability of water ices in the molecular dynamics experiment. The structures of some ices (Ih, Ic, XI, VIII, III, IX, VI, XII) turned out to be stable during molecular dynamics simulations of crystalline water ices with the PM potential at a nonzero temperature, while the structures of other ices (IV, VII) collapsed. The system consisting of one of the two identical independent water frameworks of ice VI also was stable. The stability of structures in the molecular dynamics experiment is not related to the thermodynamic stability of modeled ices because, on the one hand, some metastable ices (Ic, IX, XII) and the system consisting of one of the two identical independent frameworks of ice VI do not collapse during the computer experiment and, on the other hand, thermodynamically stable ice VII proves to be unstable.

Zero-temperature relaxation of the structures of ices IV and VII with the positions of atoms, known from the crystallography data, to the minimum of the potential energy determined by the PM potential led to insignificant shifts of all atoms (not more than several hundredths of Angstrom) without the destruction of these structures.

The cause of the destruction of ices IV and VII during molecular dynamics simulations at a nonzero temperature seems to be the inexact description of some characteristics of the interaction between water molecules by the PM potential (in particular, the use of a rigid model for water molecule) that leads to instability of these structures at a nonzero temperature.

Dynamic properties of water molecules in the structures of crystalline ices. During the study of vibrational spectra of modeled crystalline ices the regularities known from NIIS data and from other spectroscopic investigations were confirmed.

1. $\mathrm{H}_{2} \mathrm{O}$ and $\mathrm{D}_{2} \mathrm{O}$ ices with the same crystal lattice demonstrate almost the same peak frequencies of translational vibrations (the theoretical ratio is $\left.(20 / 18)^{1 / 2}: 1\right)$, and the ratio between the frequencies of librational peaks is approximately $2^{1 / 2}: 1[14,15]$.

2. The temperature increase results in broadening of vibrational spectra peaks $[14,15]$.

3. Vibrational spectra of the modifications of crystalline ices with the identical nearest environment of oxygen atoms (ices Ih and Ic) are almost identical.

4. Vibrational spectra of proton-ordered ices have sharper peaks than the spectra of proton-disordered ices; in particular, the peaks of proton-disordered ices in vibrational spectra are more blurred than the peaks in vibrational spectra of proton-ordered ices with the same oxygen framework (ices III and IX [16], Ih and XI).

Peak blurring in the vibrational spectra of proton-disordered ices is caused by that in these ices the molecules with crystallographically indiscernible oxygen atoms can be oriented in six different ways. As a result, the potential wells which they occupy, and, hence, the frequencies of their vibrations slightly differ, which results in peak blurring.

A distinction between vibrational spectra of water molecules with oxygen atoms in the same crystallographic position but with differently oriented hydrogen atoms is shown by the example of ices III [16] and XII [17]. It is clear that the environments of identically oriented water molecules can also differ, which contributes to peak blurring too.

Vibrations of molecules with oxygen atoms occupying different crystallographic positions differ not only by frequencies but also amplitudes, which follows from the distinction in the time dependence of the average square shift of the molecule mass center for these molecules.

Vibrational spectra of ice VI differ from the corresponding spectra for the system containing one of the two identical independent water frameworks constituting the ice VI structure only by a small shift of librational peaks. This circumstance indicates rather weak interaction between the independent frameworks in the ice VI structure and agrees with the stability of the system consisting of one independent water framework in the ice VI structure observed in the computer experiment.

Unlike ice VI, the interaction between independent water frameworks in ices VII and VIII appears to be essential for the stabilization of these structures: in the absence of another framework a single independent water framework of the structure of ices VII and VIII collapses during molecular dynamics simulations. In the ice VIII structure, the interaction between independent frameworks leads also to their shift relative to each other along a direction of their nonzero dipole 
moments (Fig. 1e). Moreover, the frameworks of the ice VIII structure are proton-ordered, and the separation between their molecules is larger than in the ice Ic structure. Therefore the vibrational spectra of ices VIII and Ic strongly differ.

\section{CONCLUSIONS}

Application of the molecular dynamics method has enabled us to study the dynamic properties of water molecules in many modifications of crystalline water ice. At the same time, in two cases (ices IV and VII) the structures known from the diffraction experiment collapsed, and a rather loose water framework (the density of a framework consisting of $\mathrm{H}_{2} \mathrm{O}$ molecules is $\sim 0.69 \mathrm{~g} / \mathrm{cm}^{3}$ ) occurring in real experiments only as a part of the ice VI structure (where the edges of another similar framework pass through its channels) was stable. Thus, on the one hand, if any water structure destructs during molecular dynamics simulations, it can be an artifact that does not reflect the real stability of the water structure under study. On the other hand, the stability of some input structure in the molecular dynamics experiment does not mean that it can be obtained and will be stable in the real experiment.

Disagreement between the results of molecular dynamics calculations and the data of real experiments is caused by that the potentials used insufficiently precisely describe the interaction between water molecules. Therefore the same structure can be stable in one case, and in another case it can collapse if different potentials are used in simulation. For example, in the $N P T$ ensemble with the SPC/E and TIP4P potentials the structures of ices Ih, Ic, II-IX, and XI are stable, while ice XII collapsed in simulations by the molecular dynamics method [35]. At the same time, even the quantum mechanical calculations not always lead to a correct evaluation of the stability of water structures known from experiment [31].

Unsuccessful arrangement of protons in modeled system can be one more cause of instability of particular water structures in computer experiment, although this arrangement might correspond to the Bernal-Fowler rules. For example, after simulations of proton-ordered ice XII with the TIP4P potential, only 21 structures turned out to be stable from the total 90 possible structures differing by proton arrangements in the ice XII unit cell, satisfying the Bernal-Fowler rules, and preserving the structural periodicity; all others collapsed during simulations [36].

In the molecular dynamics experiment inaccuracy in the description of interaction between water molecules by the interaction potential used can lead to the formation of the water structure that actually does not exist, while another water structure that does exist in real systems may not be formed. For example, various characteristics of the liquid-crystal phase transitions obtained by the molecular dynamics method with the SPC/E potential for some real and hypothetical ices were reported in [13]. At $100 \mathrm{~Pa}$, instead of ice $\mathrm{Ih}$, a new denser crystalline modification (not observed in real experiments) named ice B was found there as a stable crystalline phase. A distinct and stable growth of hypothetical ice B crystals at the ice $\mathrm{B} /$ melt interface was observed in simulation of the crystallization process. At the same time, no distinct growth of ice II crystals was observed at the ice II/melt interface.

Finally, the water structure formation may take a long time interval. For example, the crystallization process of ice Ih from bulk water without the initial crystallization center has recently been observed for the first time in the molecular dynamics experiment (with the TIP4P potential) in the work [10]. Here the most part of the calculation time was spent on waiting for the spontaneous crystalline embryo formation $(250 \mathrm{~ns})$; after that the whole system of 512 water molecules crystallized for $150 \mathrm{~ns}$ as the ice Ih structure. The processor time taken to calculate such a long molecular dynamics trajectories was not reported, but it is clear that computers have been calculating for many months. Thus, the time of structure formation can be rather long (in terms of the duration of computer calculations), the expected structurization of a water system in the computer experiment may not be achieved yet, but a wrong conclusion can be made that it does not occur at all.

Based on the molecular dynamics method, an approach is being developed at present to the search of crystalline water structures corresponding to the minimum of enthalpy [37]. Application of this approach has not allowed the ice II and IV structures to be obtained when the unit cell parameters were not set in advance, although the structures of other ices were obtained. In any case, this approach does not describe the formation process of one or another structure, and moreover, due to 
inaccuracy of water potentials used it can give only trial crystal structures whose existence is to be checked in real experiments. Also this approach does not yield even trial aperiodic structures.

It follows from the above that the application of the molecular dynamics method to investigate the stability of any aqueous structure not always produces correct results. Also this method not always allows correct conclusions to be made on the structurization process in water systems and on the type of structures formed. The formation and stability of any structure

in the molecular dynamics experiment does not mean its formation and stability in the real experiment, while the structure instability and the absence of structurization in the molecular dynamics experiment does not mean the structure instability and the absence of structurization in a real system.

The work was supported by the RFBR grant No. 06-03-32628.

\section{REFERENCES}

1. N. A. Bulienkov, Biofizika, 50, No. 5, 934-958 (2005).

2. M. Stoneham, Rep. Prog. Phys., 70, 1055-1097 (2007).

3. N. A. Bulienkov, Vestn. Nizhegorodskogo Un-ta im. N.I. Lobachevskogo., Ser. Fiz. Tverd. Tela, Vol 1, 19-30 (1998).

4. N. A. Bulienkov, Three Possible Branches of Determinated Modular Generalization of Crystallography, Quasicrystals and Discrete Geometry, The Fields Institute Monographs, Vol. 10, J. Patera (ed.), Providence: American Mathematical Soc., 67-134 (1998).

5. N. A. Bulienkov, Biofizika, 36, No. 2, 181-243 (1991).

6. N. A. Bulienkov and E. A. Zheligovskaya, Zh. Fiz. Khim., 80, No. 10, 1784-1805 (2006).

7. N. A. Bulienkov, Dokl. Akad. Nauk SSSR, 284, No. 6, 1392-1396 (1985).

8. C. J. Benmore, R. T. Hart, Q. Mei, et al., Phys. Rev. B, 72, No. 13, 132201(1)-132201(4) (2005).

9. G. G. Malenkov, J. Struct. Chem., 47, Supplem, S1-S31 (2006).

10. M. Matsuoto, S. Saito, and I. Ohmine, Nature, 416, 409-413 (2002).

11. E. A. Zheligovskaya and G. G. Malenkov, Usp. Khim., 75, No. 1, 64-85 (2006).

12. C. G. Salzmann, P. G. Radaelli, A. Hallbrucker, et al., Science, 311, 1758-1761 (2006).

13. L. A. Báez and P. Clancy, J. Chem. Phys., 103, No. 22, 9744-9755 (1995).

14. G. G. Malenkov, E. A. Zheligovskaya, A. A. Averkiev, et al., High Pres. Res., 17, Nos. 3-6, 273-280 (2000).

15. E. A. Zheligovskaya, G. G. Malenkov, and A. A. Averkiev, J. Struct. Chem., 42, No. 1, 7-15 (2001).

16. E. A. Zheligovskaya, ibid., 44, No. 5, 827-835 (2003).

17. E. A. Zheligovskaya and G. G. Malenkov, ibid., 46, No. 2, 276-284 (2005).

18. S. W. Peterson and H. A. Levy, Acta Crystallogr., 10, $70-76$ (1957).

19. G. P. Arnold, E. D. Finch, S. W. Rabideau, and R. G. Wenzel, J. Chem. Phys., 49, 4365-4369 (1968).

20. A. J. Leadbetter, R. C. Ward, J. W. Clark, et al., ibid., 82, No. 1, 424-428 (1985).

21. J. D. Jorgensen and T. G. Worlton, ibid., 329-333.

22. J. D. Jorgensen, R. A. Beyerlein, N. Watanabe, and T. G. Worlton, ibid., 81, No. 7, 3211-3214 (1984).

23. W. F. Kuhs, J. L. Finney, C. Vettier, and D. V. Bliss, ibid., No. 8, 3612-3623.

24. A. Rahman and F. H. Stillinger, ibid., 57, No. 9, 4009-4017 (1972).

25. V. I. Poltev, T. I. Grokhlina, and G. G. Malenkov, J. Biomol. Struct. Dyn., 2, No. 2, 413-429 (1984).

26. G. G. Malenkov, D. L. Tytik, and E. A. Zheligovskaya, J. Molec. Liq., 82, Nos. 1/2, 27-38 (1999).

27. R. J. Nelmes, J. S. Loveday, W. G. Marshall, et al., Phys. Rev. Lett., 81, No. 13, 2719-2722 (1998).

28. H. Engelhardt and B. Kamb, J. Chem. Phys., 75, No. 12, 5887-5899 (1981).

29. C. Lobban, J. L. Finney, and W. F. Kuhs, Nature, 391, 268-270 (1998).

30. S. Klotz, G. Hamel, J. S. Loveday, et al., Z. Kristallogr., 218, No. 2, 117-122 (2003).

31. M. Henry, Chem. Phys. Chem., 3, No. 7, 607-616 (2002). 
32. W. L. Vos, L. W. Finger, R. J. Hemley, and H. Mao, Phys. Rev. Lett., 71, No. 19, 3150-3153 (1993).

33. G. G. Malenkov and E. A. Zheligovskaya, J. Incl. Phenom. Macrocyclic Chem., 48, No. 1, 45-54 (2004).

34. S. L. Dong, A. I. Kolesnikov, and J. C. Li, Physica B, 263/264, 429-431 (1999).

35. A. Baranyai, A. Bartók, and A. A. Chialvo, J. Chem. Phys., 123, No. 5, 054502(1)-054502(8) (2005).

36. I. Borzsák and P. T. Cummings, Chem. Phys. Lett., 300, 359-363 (1999).

37. V. Buch, R. Martoňák, and M. Parrinello, J. Chem. Phys., 124, No. 20, 204705(1)-204705(11) (2006). 